\title{
Хроматографический анализ нуклеотидного состава секретов больших слюнных желез человека в разных возрастных периодах постнатального онтогенеза
}

\author{
(C) 2021 Чепрасова А.А. ${ }^{1}$, Попов С.C. ${ }^{1}$, Пашков А.Н. ${ }^{1}$, Обыденных Е.В. ${ }^{1}$, \\ Веревкин А.Н. ${ }^{2}$, Крыльский Е.Д. ${ }^{2}$ \\ ${ }^{1}$ Воронежский государственный медиџинский университет, Воронеж \\ ${ }^{2}$ Воронежский государственный университет, Воронеж
}

Поступила в редакцию 08.02.2021 г.

DOI: $10.17308 /$ sorpchrom.2021.21/3361

Нуклеотиды играют ключевую роль во многих биохимических и внутриклеточных процесcax, они входят в состав коферментов, принимают участие в углеводном обмене и синтезе липидов. В слюне содержатся адениловые и гуаниловые нуклеотиды, а также другие биологически активные вещества. Целью исследования явилось изучение содержания нуклеотидного состава секретов больших слюнных желез у практически здоровых доноров в различных возрастных группах.

В исследовании участвовали 60 условно здоровых добровольцев, которые были разделены на 6 возрастных групп: 1 группа - 5-8 лет, 2 группа - 9-14 лет, 3 группа - 15-19 лет, 4 группа - 20-35 лет, 5 группа - 36-60 лет, 6 группа - 61 год и выше.

Нуклеотидный состав секретов больших слюнных желез анализировали при помощи колоночной хроматографии, используя автоматизированную систему FPLS® System (Швеция). Идентификацию свободных нуклеотидов осуществляли с применением коммерческих препаратов нуклеотидов. Количество элюированных нуклеотидов определяли путем измерения площади пиков (\%).

В ходе проведенного исследования выявили, что свободные нуклеотиды в секретах больших слюнных желез элюируются в виде 6 отличных друг от друга фракций: аденозина, аденозинмонофосфата, аденозиндифосфата, гуанозиндифосфата, аденозинтрифосфата, гуанозинтрифосфата. Наибольшую площадь в секретах больших слюнных желез имеет пик, образованный экскрецией аденозиндифосфата, а минимальную - пики, образованные экскрецией гуанозиндифосфата и гуанозинтрифосфата во всех возрастных группах.

Для оценки обмена адениловых нуклеотидов рассчитан показатель «фосфатного потенциала», который отражает изменения в содержании макроэргических фосфатов у обследованных доноров. В ходе работы был определен энергетический заряд клетки для оценки энергетических процессов в организме.

Секрет слюнных желез - доступный для исследования биологический материал, который может быть использован в клинико-диагностической практике. Нуклеотидный состав секретов слюнных желез является важным показателем физиологического состояния организма, отражает энергетический обмен на разных этапах постнатального онтогенеза.

Ключевые слова: хроматографический анализ, нуклеотиды, слюнные железы, постнатальный онтогенез.

\section{Введение}

Макроэргические соединения являются источником энергии для осуществления всех физиологических функций ор- ганизма. Уникальная энергетика метаболизма у всех видов живого реализуется на основе предварительно запасенной энергии при участии аденозинтрифосфата (АТФ), который занимает централь- 
ное место среди макроэргов. Кроме того, адениловые нуклеотиды (АТФ, АДФ, АМФ) играют особую роль в регуляции обмена веществ, являясь важнейшими факторами, обеспечивающими сопряжение между процессами, генерирующими энергию и использующими ее, и тем самым связывающими различные пути обмена.

Нуклеотиды играют ключевую роль во многих биохимических и внутриклеточных процессах, они входят в состав коферментов, принимают участие в углеводном обмене и синтезе липидов. Кроме того, нуклеотиды являются компонентами активных форм витаминов, в основном группы В (рибофлавин, ниацин) [1].

Нуклеотиды являются структурными элементами рибонуклеиновой (РНК) и дезоксирибонуклеиновой (ДНК) кислот [2], они могут синтезироваться из аминокислот, но так как данные механизмы требуют больших затрат энергии, то метаболически более выгодно использовать нуклеотиды, поступающие извне с продуктами питания [3]. Нуклеиновые кислоты (ДНК, РНК), поступающие в организм в составе продуктов питания, в кишечнике расщепляются рибонуклеазами и дезоксирибонуклеазами до нуклеотидов. Затем нуклеотиды подвергаются воздействию ферментов, расщепляющих их до нуклеозидов. Последние, в свою очередь, могут подвергаться дальнейшему распаду в просвете кишки под воздействием нуклеозидаз до пуриновых и пиримидиновых оснований, но есть и другие данные о том, согласно которым, нуклеозиды всасываются в неизмененном состоянии, и последующий процесс их превращения происходит в структуре энтероцитов [4]. Около 5\% нуклеозидов может транспортироваться к разным органам, образовывая тканевой пул (в первую очередь в печени, тонкой кишке и мышечной ткани). В тонкой кишке всасывается около 90\% нуклеотидов, лишь небольшое их количество достигает толстой кишки и оказывает пре- биотическое действие, метаболизируясь представителями кишечного микробиоценоза [5].

При патологических состояниях (гипоксия, ишемия, воспаление и др.) из различных клеток во внеклеточное пространство высвобождаются нуклеотиды [6]. В слюне содержатся адениловые и гуаниловые нуклеотиды, а также другие биологически активные вещества [7].

Целью исследования явилось изучение содержания нуклеотидов в секретах больших слюнных желез (БСЖ) у практически здоровых доноров в различных возрастных группах.

\section{Экспериментальная часть}

Проведено исследование секретов БСЖ у 60 практически здоровых людей. Обследуемые люди были разделены на 6 возрастных групп: 1 группа - 5-8 лет, 2 группа - 9-14 лет, 3 группа - 15-19 лет, 4 группа - 20-35 лет, 5 группа - 36-60 лет, 6 группа - 61 год и выше. Формирование возрастных групп для исследования основывалось на периодизации постнатального онтогенеза человека. Работа одобрена этическим комитетом ВГМУ им Н.Н. Бурденко (протокол от 27.02.2014 г. №1). Перед проведением клинического исследования у всех доноров получено информированное согласие в соответствии с принципами, изложенными в Хельсинкской Декларации Всемирной медицинской ассоциации (64th WMA General Assembly, Fortaleza, Brazil, October 2013) и Федеральном законе Российской Федерации № 323-Ф3 от 21.11.2011 г. «Об основах охраны здоровья граждан в Российской Федерации».

Секреты больших слюнных желез левой (ЛОУЖ) и правой (ПОУЖ) околоушных, подчелюстных и подъязычных (ПЯЖ+ПЧЖ) слюнных желез - собирали утром натощак при помощи слюносборника (Sarstedt D-51588 Numbrecht), в течение 10 минут стоматологические тампоны держали в ротовой полости. Доноры молчали, дышали через нос. Пробы 
секретов БСЖ центрифугировали 10 минут при 3000 об/мин., полученный центрифугат использовали в исследовании. К 600 мкл надосадочной жидкости добавляли 400 мкл холодного раствора $\mathrm{HClO}_{4}$, хорошо взбалтывали и центрифугировали. Из центрифугата отбирали 600 мкл раствора и добавляли 8 капель $\mathrm{K}_{2} \mathrm{CO}_{3}$, перемешивали, $\mathrm{pH}$ полученного раствора должен быть нейтральным. Далее повторяли центрифугирование при 3000 об/мин, из полученного центрифугата отбирали пробу объемом 200 мкл.

Для исследования адениловых и гуаниловых нуклеотидов в слюне использовался хроматографический метод с использованием автоматизированной системы FPLS System (Швеция) на колонке «Q».

Предварительно проводили промывку дистиллированной водой, далее проходило уравновешивание буферным раствором. Для уравновешивания использовалось 2 буфера: буфер А - 10-2n $\mathrm{HCl}$ $(\mathrm{pH}=7.5)$, буфер $\mathrm{B}-10-2 \mathrm{n} \mathrm{HCl}+0.5 \mathrm{M}$ $\mathrm{NaCl}(\mathrm{pH}=8.5)$. Элюирование происходило со скоростью $1.5 \mathrm{~cm}^{3} /$ мин. Регистрация происходила при длине волны 254 нм.

Идентификацию свободных нуклеотидов осуществляли с применением коммерческих препаратов нуклеотидов. Количество элюированных нуклеотидов определяли путем измерения площади пиков (\%).

Для оценки обмена адениловых нуклеотидов может быть использован показатель «фосфатного потенциала», который хорошо отражает изменения в содержании макроэргических фосфатов у обследованных доноров. «Фосфатный потенциал» характеризуется соотношением АДФ×АМФ/АТФ [8].

Уровень содержания АТФ, а также соотношение компонентов фракции адениловых нуклеотидов оказывает определяющее влияние на характер, интенсивность и пути ресинтеза АТФ и метаболизма в целом, поэтому анализ динамики содержания макроэргических соедине- ний в тканях является одной из важных задач при изучении энергетических процессов в организме. Энергетический метаболизм обеспечивает взаимопревращение между АТФ, АДФ и АМФ, определяя энергетический заряд клетки (АТФ+0.5АДФ)/(АТФ+АДФ+АМФ).

Статистическую обработку данных проводили, используя методы математической и медицинской статистики при помощи пакета анализа данных Microsoft Office Excel и с помощью статистического пакета STADIA 7.0(InCo, Россия). Результаты выражали в виде $\mathrm{M} \pm \mathrm{m}$, где $\mathrm{M}-$ средняя арифметическая величина, а $\mathrm{m}$ ошибка средней величины. Для выявления значимых различий между независимыми группами использовали двухвыборочный t-тест Стьюдента. При проверке статистических гипотез принимался $5 \%$ уровень значимости.

\section{Обсуждение результатов}

При изучении полученных хроматограмм было установлено наличие в секретах БСЖ следующих нуклеотидов: АТФ, АДФ, АМФ, ГТФ, ГДФ, а также нуклеозида аденозина.

Из рисунка 1 видно, что наибольшая концентрация аденозина в секрете ПОУЖ приходится на 1 возрастную группу (5-8 лет), далее происходит постепенное снижение с минимальным значением в 4 возрастной группе (20-35 лет). С увеличением возраста концентрация аденозина постепенно возрастает, притом значения в 6 возрастной группе (61 лет и выше) практически достигают значений концентрации в 3 возрастной группы (15-19 лет). Концентрация АМФ в первых двух возрастных группах (5-8 и 9-14 лет) находится на одном уровне, далее происходит резкий рост количества данного вещества в 3 и 4 группах (15-19 и 20-35 лет). В возрастном периоде 36-60 лет значение концентрации АМФ соответствует значениям первых двух групп (5-8 и 9-14 лет). У доноров, имеющих возраст 61 и более лет, происходит рост содержания данного нук- 


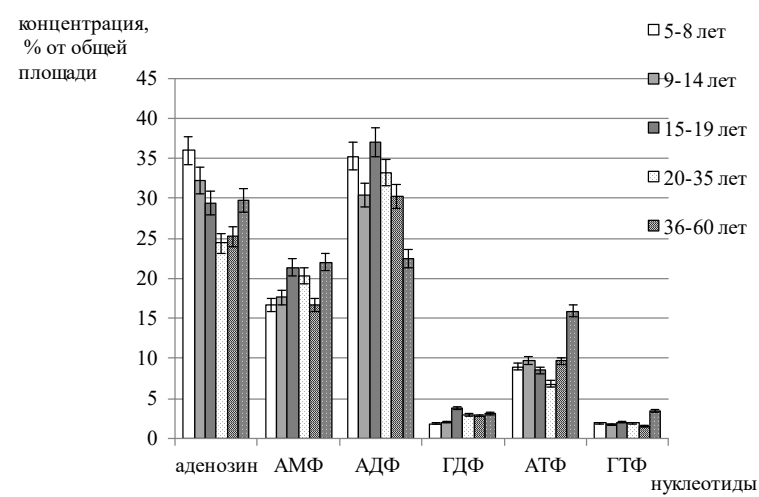

Рис 1. Нуклеотиды в секрете правой околоушной железы в разные возрастные периоды онтогенеза

Fig 1. Nucleotides in the secretion of the right parotid gland in different age groups of ontogenesis

леотида, при этом значения концентрации статистически не отличаются от значений 3 и 4 возрастных групп $(\mathrm{p} \geq 0,05)$. Наибольшая концентрация АДФ характерна для возраста 5-8 лет, далее происходит резкое снижение в группе доноров 9-14 лет. С увеличением возраста происходит постепенное повышение концентрации АДФ, значения 3 возрастной группы статистически не отличаются от значений 1 возрастной группы ( $\mathrm{p} \geq 0,05)$. У доноров 4 возрастной группы наблюдается постепенное падение концентрации АДФ с минимальным значением

в 6 возрастной группе. Содержание ГДФ в секрете ПОУЖ невелико. В 1 возрастной группе имеет минимальное значение, далее происходит плавное увеличение концентрации нуклеотида с максимальным значением концентрации ГДФ в 3 возрастной группе. В 4, 5 и 6 возрастных группах наблюдается небольшое снижение концентрации изучаемого нуклеотида, данные значения статистически не отличаются ( $\mathrm{p} \geq 0.05)$. Концентрация АТФ в секрете ПОУЖ имеет более высокие показатели, чем концентрация ГДФ. В возрастных группах, соответствующих возрасту 5-14 лет уровень АТФ одинаков, далее происходит резкое снижение содержания данного нуклеотида с минимальным значением в 4 возрастной группе. С возрастом, после 35

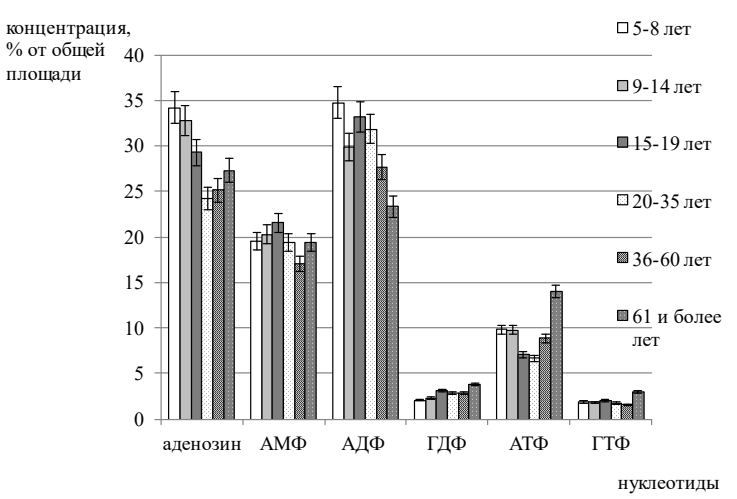

Рис. 2. Нуклеотиды в секрете левой околоушной железы в разные возрастные периоды

Fig. 2. Nucleotides in the secretion of the left parotid gland in different age groups

лет происходит значительный рост концентрации АТФ с максимальным значением в 6 возрастной группе (61 и более лет). Гуаниловый нуклеотид - ГТФ, так же, как и ГДФ в секрете ПОУЖ содержится в незначительном количестве. В возрасте от 5 до 35 лет значения концентрации практически не изменяются, а к 36 годам происходит небольшое снижение. Максимальное значение концентрации ГТФ характерно для 6 возрастной группы, что соответствует возрасту 61 и более лет.

Как следует из рисунка 2, изменение концентрации нуклеозида аденозина в секрете ЛОУЖ соответствует тому профилю, который наблюдается в секрете ПОУЖ. То есть, наивысшая концентрация аденозина приходится на 1 возрастную группу (5-8 лет), а далее концентрация снижается с минимальным значением в 4 возрастной группе (20-35 лет). С возрастом количество аденозина постепенно увеличивается, притом значения в 6 возрастной группе (61 лет и выше) практически достигают значений концентрации в 3 возрастной группы (15-19 лет) ( $\geq 00,05)$. В отличие от секрета ПОУЖ профиль значений концентрации АМФ в секрете ЛОУЖ имеет свои особенности. А именно, в первых четырех возрастных группах (5-35 лет) содержание нуклеотида находится на одном уровне, а в 5 группе (36-60 лет) про- 


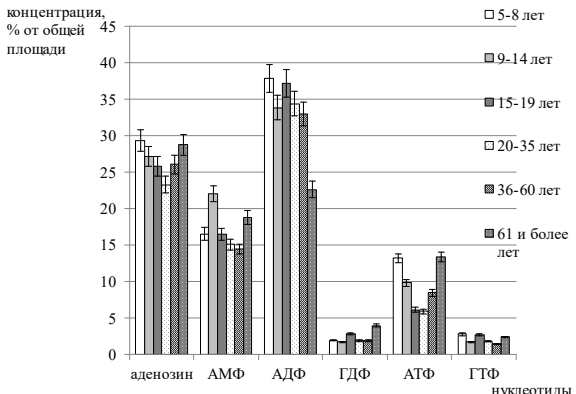

Рис. 3. Нуклеотиды в секретах подъязычной с подчелюстных желез в разные возрастные периоды

Fig. 3. Nucleotides in the secretions of the sublingual from the submandibular glands in different age groups

исходит небольшое снижение концентрации АМФ. В возрастной группе участников 61 и более лет значения концентрации АМФ равнозначны значениям первых четырех групп ( $\mathrm{p} \geq 0.05)$. Особенности изменения содержания нуклеотида АДФ в секрете ЛОУЖ соответствует профилю изменений концентрации в секрете ПОУЖ. Высокие значения концентрации наблюдаются в возрастной группе

5-8 лет, далее происходит резкое уменьшение содержания АДФ в секрете доноров 9-14 лет. Значения 3 возрастной группы статистически не отличаются от значений 1 возрастной группы ( $\geq 00,05)$. У доноров 4 возрастной группы наблюдается постепенное падение концентрации АДФ с минимальным значением в 6 возрастной группе (61 и более лет). Содержание ГДФ в секрете ЛОУЖ невелико. В 1 возрастной группе имеет минимальное значение, далее происходит плавное увеличение концентрации нуклеотида с максимальным значением концентрации ГДФ в 6 возрастной группе. Концентрация АТФ в секрете ЛОУЖ имеет более высокие значения, чем концентрация ГДФ. В возрастных группах, соответствующих возрасту 5-14 лет уровень АТФ одинаков, далее происходит резкое снижение содержания данного нуклеотида с минимальным значением в 4 возрастной группе (20-35 лет). С возрастом, после 35 лет происходит значи-

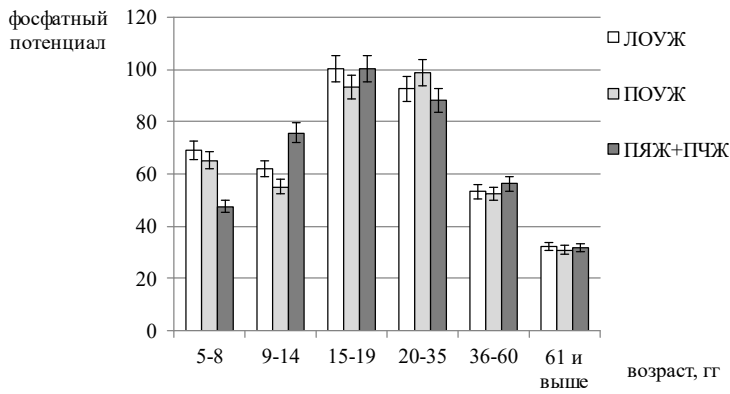

Рис. 4. Фосфатный потенциал секретов

больших слюнных желез человека в постнатальном онтогенезе

Fig. 4. Phosphate potential of secretions of human major salivary glands in postnatal ontogenesis

тельный рост концентрации АТФ с максимальным значением в 6 возрастной группе (61 и более лет). Гуаниловый нуклеотид ГТФ в секрете ЛОУЖ содержится также в незначительном количестве. В возрасте от 5 до 35 лет значения концентрации практически не изменяются, а к 36 годам происходит небольшое снижение. Максимальное значение концентрации ГТФ характерно для 6 возрастной группы, что соответствует возрасту 61 и более лет.

Из рисунка 3 наглядно прослеживается следующая ситуация по нуклеотидному составу секретов ПЯЖ+ПЧЖ. Наибольшее содержание нуклеозида аденозина характерно для 1 и 6 возрастных групп (5-8 и 61 и более лет), а минимальное значение установлено для 4 возрастной группы (20-35 лет). Изменение концентрации АМФ в секретах ПЯЖ+ПЧЖ в различных возрастных периодах постнатального онтогенеза, в отличие от секретов околоушных желез, имеет некоторые отличия. Во-первых, максимальное содержание данного нуклеотида характерно для 2 возрастной группы, а минимальное для 4 и 5 групп доноров. Во-вторых, значения концентраций в секретах в 1 и 3 возрастных групп статистически не отличаются ( $\mathrm{p} \geq 0$ ю05). Но есть и небольшое сходство c особенностями содержания АМФ в секретах околоушных слюнных желез, а именно, в возрастной группе 61 и более 
лет происходит второй рост концентрации нуклеотида в секрете данной слюнной железы. Профиль содержания АДФ в секрете ПЯЖ+ПЧЖ полностью повторяет профиль значений концентрации изучаемого нуклеотида в секретах ПОУЖ и ЛОУЖ. Изменение концентрации ГДФ в секрете ПЯЖ+ПЧЖ имеет те же характеристики, что и в секретах ЛОУЖ. Но для секрета данной железы характерно более четкое формирование двух максимумов содержания нуклеотида: в 3 и 6 возрастных группах. В остальных группах значения концентрации ГДФ статистически не отличаются $(\mathrm{p} \geq 0.05)$. Изменение концентрации АТФ в секрете ПЯЖ+ПЧЖ соответствует профилю значений концентрации в секрете ЛОУЖ. Нуклеотид ГДФ в секрете ПЯЖ+ПЧЖ содержится также в незначительном количестве. В возрасте от 5-8 лет и 15-19 лет содержание данного нуклеотида находится на одном уровне, а значения в возрастных периодах 9-14 и 20-35 лет уже чуть снижены, минимальное значение характерно для возраста 36-60 лет. В возрастном периоде 61 и более лет происходит повторное возрастание концентрации нуклеотида ГТФ.

При рассмотрении рисунков 1, 2 и 3 можно сравнить значения концентрации нуклеотидов в секретах БСЖ. Содержание нуклеозида аденозина в секретах ЛОУЖ и ПОУЖ находится на одном уровне, а в секретах ПЯЖ+ПЧЖ показания значительно ниже. Данная картина характерна для первых трёх возрастных групп (5-8, 9-14, 15-19 лет). Начиная с возраста 20 лет и старше, концентрация аденозина в секретах всех трёх БСЖ в каждом данном возрастном периоде статистически не отличается. Что касается концентрации АМФ в секретах разных БСЖ в 1 возрастной группе, то в секрете ЛОУЖ концентрация нуклеотида достоверно выше, чем в секретах ПЯЖ+ПЧЖ и ПОУЖ ( $\geq \geq 0.05)$. В 2 возрастной группе значения концентрации в секретах ЛОУЖ и ПЯЖ +ПЧЖ статистически не отличаются $(\mathrm{p} \geq 0.05)$, но значительно больше, чем в ПОУЖ. В 3, 4 и 5 возрастных группах концентрация данного нуклеотида в секретах ЛОУЖ и ПОУЖ находятся на одном уровне, а в секретах ПЯЖ+ПЧЖ значения концентрации ниже. В группе 61 и более лет наибольшая концентрация АМФ наблюдается в ПОУЖ, а в ЛОУЖ и ПЯЖ+ПЧЖ концентрация приблизительно равна ( $\mathrm{p} \geq 0.05)$. Нуклеотид АДФ в возрастном периоде 58 лет содержится в одинаковом количестве в секретах БСЖ ( $\mathrm{p} \geq 0.05)$. В группе доноров 9-14 лет наибольшая концентрация характерна для секретов ПЯЖ+ПЧЖ, а наименьшая в секретах ЛОУЖ и ПОУЖ, в которых количество нуклеотида находится на одном уровне $(\mathrm{p} \geq 0.05)$. В 3 возрастной группе (1519 лет) количество АДФ в секретах ПОУЖ и ПЯЖ+ПЧЖ одинаково ( $\mathrm{p} \geq 0.05)$, а в секрете ЛОУЖ наблюдается меньшая концентрация. В возрастной группе 2035 лет количество АДФ в секретах БСЖ статистически не отличается ( $\mathrm{p} \geq 0.05)$. В 5 возрастной группе наименьшая концентрация нуклеотида характерна для секрета ЛОУЖ, а содержание АДФ в секретах ПОУЖ и, особенно, ПЯЖ+ПЧЖ возрастает. Для возрастной группы 61 и более лет характерна одинаковая концентрация данного нуклеотида в секретах всех БСЖ $(\mathrm{p} \geq 0.05)$.

В 1 возрастной группе (5-8 лет) наибольшая концентрация ГДФ характерна для ЛОУЖ, а наименьшая для ПОУЖ. В 2 возрастной группе (9-14 лет) уже происходит резкое изменение: в ЛОУЖ содержится большее количество ГДФ, чем в ПОУЖ и, особенно, в ПЯЖ+ПЧЖ. В возрастном периоде 1519 лет максимальное содержание данного нуклеотида характерно для ПОУЖ, а минимальное в ПЯЖ+ПЧЖ. В 4 и 5 возрастных периодах в ЛОУЖ и ПОУЖ одинаковая концентрация ГДФ ( $\mathrm{p} \geq 0.05)$, а наименьшая концентрация характерна для секрета ПЯЖ+ПЧЖ. В возрасте 61 и более лет самая высокая концентрация ГДФ в секретах всех больших слюнных желез, но в секретах ЛОУЖ и 


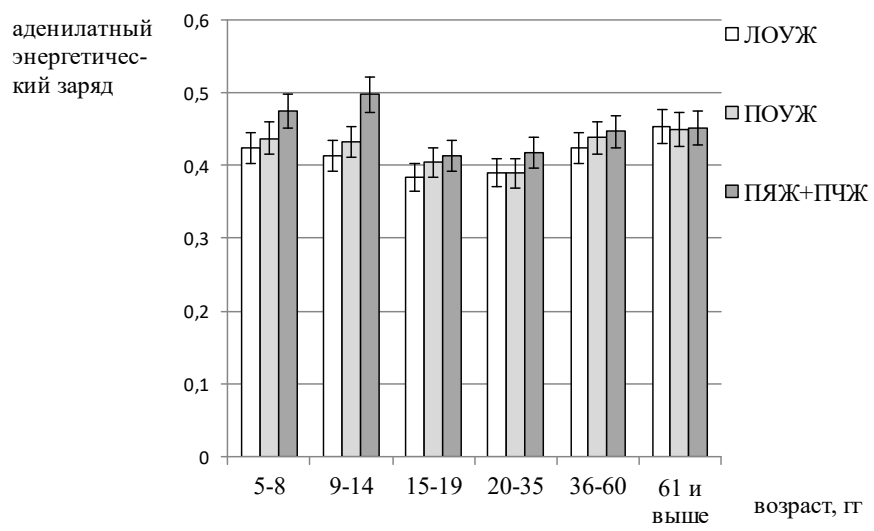

Рис. 5. Аденилатный энергетический заряд секретов больших слюнных желез человека в постнатальном онтогенезе

Fig. 5. Adenylate energy charge of secretions of human major salivary gland in postnatal ontogenesis

ПЯЖ+ПЧЖ содержание данного нуклеотида одинаково ( $\mathrm{p} \geq 0.05)$, а в ПОУЖ имеет место меньшее количество. При характеристике содержания АТФ в секретах БСЖ складывается следующая картина: в 1 возрастной группе лидирует по содержанию АТФ ПЯЖ+ПЧЖ, в ЛОУЖ и ПОУЖ концентрация нуклеотида ниже. В группе 9-14 лет секреты всех больших слюнных желез характеризуются одинаковым уровнем концентрации АТФ ( $\mathrm{p} \geq 0.05)$. Начиная с возраста 15 лет, концентрация в секретах ПЯЖ+ПЧЖ во всех возрастных группах имеет минимальное значение, притом сначала лидирует по содержанию АТФ ПОУЖ (15-19 лет), далее ЛОУЖ и ПОУЖ равнозначны (20-35 лет), а в 6 возрастной группе ПОУЖ опять имеет максимальное значение концентрации АТФ. Количество ГТФ в секретах всех БСЖ не высоко. Первая возрастная группа (5-8 лет) характеризуется максимальной концентрацией в секрете ПЯЖ+ПЧЖ, а минимальное количество - в ЛОУЖ и ПОУЖ ( $\mathrm{p} \geq 0.05)$. В группе участников 914 лет концентрация ГТФ в секретах всех БСЖ статистически не отличается $(\mathrm{p} \geq 0.05)$. В 3 возрастной группе наблюдается такой же профиль значений, как и в 1 группе. В 4 и 5 группах (20-35 и 3660 лет), как и в 2 возрастной группе (914 лет) значения концентрации нуклеотида ГТФ в секретах всех БСЖ одинаковы $(p \geq 0.05)$. В группе 61 и более лет максимальная концентрация ГТФ характерна для секрета ПОУЖ, а минимальная - для секрета ПЯЖ+ПЧЖ.

При определении «фосфатного потенциала» для каждой слюнной железы в разные возрастные периоды были получены данные, отраженные в рисунке 4. В секретах ЛОУЖ и ПОУЖ в 2 возрастной группе происходит небольшое снижение данного показателя обмена адениловых нуклеотидов. Начиная с 15 летнего возраста до 35 лет, происходит увеличение фосфатного потенциала, в пятой возрастной группе (36-60 лет) снижение, а минимум данного показателя приходится на возраст 61 и более лет. В секретах ПЯЖ+ПЧЖ есть некоторые отличия от ЛОУЖ и ПОУЖ. Начиная с 5 летнего возраста, происходит постепенное увеличение фосфатного потенциала, но в 5 возрастной группе (36-60 лет), также как и в секретах околоушных желез, происходит резкое снижение данного показателя с минимальным значением в 6 возрастном периоде (61 и более лет). При сравнении значений фосфатного потенциала между секретами различных БСЖ можно пронаблюдать следующую ситуацию. В 1 возрастной группе показатель обмена адениловых нуклеотидов в секретах околоушных желез находятся на одном уровне ( $\mathrm{p} \geq 0.05)$, данные значения значительно выше, чем в секретах подъязычной и подчелюстной слюнных желез. Во 2 возрастной группе максималь- 
ное значение фосфатного потенциала характерно для секрета ПЯЖ+ПЧЖ, а минимальное для ПОУЖ. В 3, 4, 5 и 6 возрастных групп значения фосфатного потенциала между секретами БСЖ статистически не отличаются ( $\mathrm{p} \geq 0.05)$.

Особенности изменения аденилатного энергетического заряда (АЭЗ) в секретах больших слюнных желез были выявлены при анализе рисунка 5. Установлено, что в 3 и 4 возрастных группах (15-19 и 2035 лет) установлены низкие значения аденилатного энергетического заряда. При сравнении значений АЭЗ секретов больших слюнных желез в каждом возрастном периоде, было установлено, что в 1 и 2 возрастных группах данный показатель в секрете подъязычной с подчелюстной слюнных желез превышает значения секретов околоушных желез. Начиная с 15 летнего возраста, в каждой возрастной группе значения АЭЗ в секретах больших слюнных желез статистически не отличаются ( $\mathrm{p} \geq 0.05)$.

\section{Список литературы}

1. Кешишян Е.С., Бердникова Е.К. // Лечаший врач. 2004. № 1. С. 53-54.

2. Ohyanagi H., Nishimatsu S., Kanbara Y. et al. // J. Parenter Enteral Nutr. 1989. Vol. 13. No 1. pp. 51-58.

3. Carver J.D. // Am. J. Clin. Nutrition. 2003. Vol. 77. No 6. pp. 1550-1554.

4. Uauy R. Dietary nucleotides and requirements in early life. New York. Raven Press. 1989. pp. 265-280.

\section{Заключение}

Таким образом, в ходе исследования нами установлено наличие в секретах больших слюнных желез следующих нуклеотидов: АТФ, АДФ, АМФ, ГТФ, ГДФ, а также нуклеозида аденозина. Можно отметить, что наименьшая концентрация в секретах слюнных желез характерна для нуклеотидов ГТФ и ГДФ, а наибольшая - для нуклеозида аденозина и для нуклеотида АДФ. Нуклеотидный состав секретов слюнных желез может быть важным показателем физиологического состояния организма. Значения концентрации нуклеотидов в секретах больших слюнных желез в одинаковых возрастных группах различны, кроме того, было зафиксировано изменение содержания нуклеотидов в секретах слюнных желез в различных возрастных группах. Полученные результаты могут быть использованы в клиникодиагностической практике в виде критериев энергетического обмена, как защитного механизма по поддержанию гомеостаза организма при стрессе.

5. Singhal A., Macfarlane G., Macfarlane S. et al. // Am. J. Clin. Nutrition. 2008. Vol. 87. No 6. pp. 1785-1792.

6. Eltzschig H.K., Sitkovsky M.V., Robson S.C. // N Engl J Med. 2012. No 367. pp. 23222333.

7. Мячина О.В., Зуйкова А.А., Пашков А.Н. // Вестник новых медицинских технологий. 2017. Т. 24. № 4. С. 85-88.

8. Прохоров М.Ю., Тиунов М.П., Шакалис Д.А. // Лабораторное дело. 1977. № 9. С. 535-536.

\title{
Chromatographic analysis of the nucleotide composition of the secretions of the large human salivary glands at different age periods of postnatal ontogenesis
}

\author{
(C) 2021 Cheprasova A.A. ${ }^{1}$, Popov S.S. ${ }^{1}$, Pashkov A.N. ${ }^{1}$, Obydennykh E.V. ${ }^{1}$, \\ Verevkin A.N. ${ }^{2}$, Krylsky E.D. ${ }^{2}$ \\ ${ }^{I}$ Voronezh State Medical University named after N.N. Burdenko, Voronezh, Russian Federation \\ ${ }^{2}$ Voronezh State University, Voronezh, Russian Federation
}

Nucleotides play a key role in many biochemical and intracellular processes. They are coenzymes and are involved in the carbohydrate metabolism and lipid synthesis. Saliva contains adenyl and guanyl nu- 
cleotides, as well as other biologically active substances. The aim of the study was the investigation of the content of the nucleotide composition of the secretion of the major salivary glands in practically healthy donors of different age groups. The study involved 60 apparently healthy volunteers who were divided into 6 age groups: Group 1 - 5-8 years old, Group 2-9-14 years old, Group 3-15-19 years old, Group 4-20-35 years old, Group 5 - 36-60 years old, Group 6 - 61 years old and above.

The nucleotide composition of the saliva of the major salivary glands was analysed by column chromatography using an automated FPLS ${ }^{\circledR}$ System (Sweden). The identification of free nucleotides was carried out using commercial nucleotide preparations. The amount of eluted nucleotides was determined by measuring the area of the peaks $(\%)$.

The study revealed that the free nucleotides in the secretions of the major salivary glands are eluted in the form of 6 different fractions: adenosine, adenosine monophosphate, adenosine diphosphate, guanosine diphosphate, adenosine triphosphate, and guanosine triphosphate. The largest area in the secretions of the major salivary glands has a peak formed by the excretion of adenosine diphosphate, and the minimum is the peaks formed by the excretion of guanosine diphosphate and guanosine triphosphate in all age groups.

For the assessment of the metabolism of adenyl nucleotides, the index of "phosphate potential" was calculated, which reflects changes in the content of high-energy phosphates in the examined donors. Over the course of the study, the energy charge of the cell was determined for the assessment of the energy processes in the body.

The secretion of the salivary glands is a biological material available for research, which can be used in clinical and diagnostic practices. The nucleotide composition of the secretions of the salivary glands is an important indicator of the physiological state of the body, reflecting energy metabolism at different stages of postnatal ontogenesis.

Keywords: chromatographic analysis, nucleotides, salivary glands, postnatal ontogenesis.

\section{References}

1. Keshishyan E.S., Berdnikova E.K., Lechashchij vrach, 2004, No 1, pp. 53-54.

2. Ohyanagi H., Nishimatsu S., Kanbara Y. et al., J. Parenter Enteral Nutr., 1989, Vol. 13, No 1, pp. 51-58.

3. Carver J.D., Am. J. Clin. Nutrition, 2003, Vol. 77, No 6, pp. 1550-1554.

4. Uauy R., Dietary nucleotides and requirements in early life, New York, Raven Press, 1989, pp. 265-280.

Чепрасова Анна Александровна - ассистент кафедры биологии, Воронежский государственный медицинский университет им. Н.Н. Бурденко, Воронеж

Попов Сергей Сергеевич - д.м.н., зав. кафедрой организации фармацевтического дела, клинической фармации и фармакогнозии, доцент, Воронежский государственный медицинский университет имени Н.Н. Бурденко, Воронеж

Пашков Александр Николаевич - заведующий кафедрой биологии, д.б.н., профессор, Воронежский государственный медицинский университет имени Н.Н. Бурденко, Воронеж

Обыденных Екатерина Викторовна - инженер кафедры биологии, Воронежский государственный медицинский университет имени Н.Н. Бурденко, Воронеж

Веревкин Алексей Николаевич - к.б.н., старший преподаватель кафедры медицинской биохимии и микробиологии, Воронежский государственный университет, Воронеж

Крыльский Евгений Дмитриевич - к.б.н., доцент кафедры медицинской биохимии и микробиологии, Воронежский государственный университет, Воронеж
5. Singhal A., Macfarlane G., Macfarlane S. et al., Am. J. Clin. Nutrition, 2008, Vol. 87, No 6, pp. 1785-1792.

6. Eltzschig H.K., Sitkovsky M.V., Robson S.C., $N$ Engl J Med., 2012, No 367, pp. 23222333.

7. Myachina O.V., Zujkova A.A., Pashkov A.N., Vestnik novyh medicinskih tekhnologij, 2017, Vol. 24, No 4, pp. 85-88.

8. Prohorov M.YU., Tiunov M.P., SHakalis D.A., Laboratornoe delo, 1977, No 9, pp. 535-536.

Cheprasova Anna A. - Assistant of the Department of Biology, Voronezh State Medical University named after N.N. Burdenko, Voronezh

Popov Sergey S. - Head of the Department of Organization of Pharmaceutical Business, Clinical Pharmacy and Pharmacognosy, Doctor of Medical Sciences, Docent, Voronezh State Medical University named after N.N. Burdenko, Voronezh

Pashkov Alexander N. - Head of the Department of Biology, Doctor of Biological Sciences, Professor, Voronezh State Medical University named after N.N. Burdenko, Voronezh

Obydennykh Ekaterina V. - Engineer, Department of Biology, Voronezh State Medical University named after N.N. Burdenko, Voronezh

Verevkin Alexey N. - Ph.D., Senior Lecturer, Department of Medical Biochemistry and Microbiology, Voronezh State University, Voronezh

Kryl'skii Evgenii D. - Ph.D., Docent of the Department of Medical Biochemistry and Microbiology, Voronezh State University, Voronezh 